\title{
On Zagreb Eccentricity Indices
}

\author{
Rundan Xing, ${ }^{a}$ Bo Zhou, ${ }^{a, *}$ and Nenad Trinajstić \\ ${ }^{a}$ Department of Mathematics, South China Normal University, Guangzhou 510631, China \\ ${ }^{\mathrm{b}}$ The Rugjer Bošković Institute, P. O. Box 180, HR-10 002 Zagreb, Croatia
}

RECEIVED NOVEMBER 20, 2010; REVISED JUNE 1, 2011; ACCEPTED JUNE 2, 2011

\begin{abstract}
Zagreb eccentricity indices were proposed analogously to Zagreb indices already known and used for almost forty years. For a connected graph, the first Zagreb eccentricity index is defined as the sum of the squares of the eccentricities of the vertices, and the second Zagreb eccentricity index is defined as the sum of the products of the eccentricities of pairs of adjacent vertices. We report mathematical properties, especially lower and upper bounds of trees and general graphs in terms of graph invariants and the corresponding extremal graphs, Nordhaus-Gaddum-type results, and the ordering of trees with small and large Zagreb eccentricity indices. (doi: 10.5562/cca1801)
\end{abstract}

Keywords: Zagreb indices, Zagreb eccentricity indices, lower and upper bounds, graph invariants, trees, general graphs

\section{INTRODUCTION}

Let $G$ be a connected graph with vertex set $V(G)$ and edge set $E(G)$. For a vertex $u \in V(G), e_{G}(u)$ or $e_{u}$ denotes the eccentricity of $u$ in $G$, which is the length of a path from $u$ to a vertex $v$ that is farthest from $u$, i.e., $e_{u}=\max \{d(u, v \mid G): v \in V(G)\}$, where $d(u, v \mid G)$ denotes the distance between $u$ and $v$ in $G{ }^{1}$

The Zagreb eccentricity indices are introduced in an analogy with the Zagreb indices ${ }^{2-4}$ by replacing the vertex degrees with the vertex eccentricities. Thus, the first Zagreb eccentricity index of a graph $G$ is defined as:

$$
\xi_{1}=\xi_{1}(G)=\sum_{u \in V(G)} e_{u}^{2},
$$

and the second Zagreb eccentricity index of $G$ is defined as:

$$
\xi_{2}=\xi_{2}(G)=\sum_{u v \in E(G)} e_{u} e_{v} .
$$

Note that degrees are "local properties", while eccentricities are "global properties" of the vertices.

These two indices are recently introduced by Vukičević and Graovac. ${ }^{5}$ However, it should be pointed out that there were a number of eccentricity-based molecular descriptors already proposed in the literature, ${ }^{6,7}$ such as the eccentric connectivity index ${ }^{8,9}$ (see References 10-12 for recent results) and the eccentric adjacency index. ${ }^{13}$ Vukičević and Graovac have shown in their paper ${ }^{5}$ that $\frac{\xi_{1}(G)}{|V(G)|} \geq \frac{\xi_{2}(G)}{|E(G)|}$ if $G$ is a tree or a unicyclic graph but is not true for bicyclic graphs.

In the present paper, we give lower and upper bounds for the first and the second Zagreb eccentricity indices of $n$-vertex trees with fixed diameter, and lower bounds for the first and the second Zagreb eccentricity indices of $n$-vertex trees with fixed matching number, and characterize the extremal cases, and determine the $n$-vertex trees with respectively the minimum, secondminimum and third-minimum, as well as the maximum, second-maximum and third-maximum indices $\xi_{1}$ and $\xi_{2}$ for $n \geq 6$. We also give lower and upper bounds for the first and the second Zagreb eccentricity indices of connected graphs in terms of graph invariants such as the number of vertices, the number of edges, the radius and the diameter, and give the Nordhaus-Gaddum-type results. ${ }^{14}$

\section{PRELIMINARIES}

For a connected graph $G$, the radius $r(G)$ and the diameter $D(G)$ are, respectively, the minimum and maximum eccentricities among the vertices of $G .^{1}$ A connected graph is called a self-centered graph if all of its vertices have the same eccentricity. Evidently, a connected graph $G$ is self-centered if and only if $r(G)=D(G)$.

\footnotetext{
* Author to whom correspondence should be addressed. (E-mail: zhoubo@scnu.edu.cn)
} 


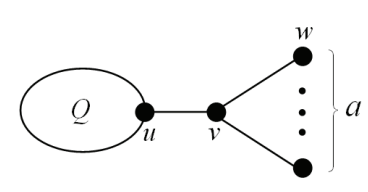

$G_{1}$

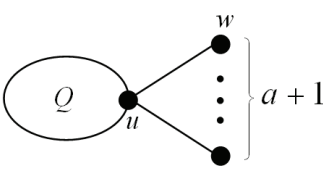

$G_{2}$
Figure 1. The trees $G_{1}$ and $G_{2}$ in Lemma 1.

Let $K_{n}$ be the $n$-vertex complete graph. Let $S_{n}$ and $P_{n}$ be the $n$-vertex star and path, respectively. By direct calculation, $\xi_{1}\left(K_{n}\right)=n$ and $\xi_{2}\left(K_{n}\right)=\frac{n(n-1)}{2}$ for $n \geq 2, \quad \xi_{1}\left(S_{n}\right)=4 n-3$, and $\xi_{2}\left(S_{n}\right)=2(n-1)$ for $n \geq 3$, and if $n \geq 2$ is even, then:

$$
\begin{aligned}
\xi_{1}\left(P_{n}\right)= & 2 \sum_{i=n / 2}^{n-1} i^{2}=2\left(\sum_{i=1}^{n-1} i^{2}-\sum_{i=1}^{n / 2-1} i^{2}\right) \\
= & \frac{1}{3}(n-1) n(2 n-1)-\frac{1}{3}\left(\frac{n}{2}-1\right) \cdot \frac{n}{2} \cdot(n-1) \\
= & \frac{7 n^{3}-9 n^{2}+2 n}{12} \\
\xi_{2}\left(P_{n}\right)= & 2 \sum_{i=n / 2}^{n-2} i(i+1)+\left(\frac{n}{2}\right)^{2} \\
= & 2\left(\sum_{i=1}^{n-2} i^{2}-\sum_{i=1}^{n / 2-1} i^{2}+\sum_{i=n / 2}^{n-2} i\right)+\frac{n^{2}}{4} \\
= & \frac{1}{3}(n-2)(n-1)(2 n-3)-\frac{1}{3}\left(\frac{n}{2}-1\right) . \\
& \frac{n}{2} \cdot(n-1)+\left(\frac{n}{2}-1\right)\left(\frac{3 n}{2}-2\right)+\frac{n^{2}}{4} \\
= & \frac{7 n^{3}-21 n^{2}+20 n}{12},
\end{aligned}
$$

and if $n \geq 3$ is $o d d$, then:

$$
\begin{aligned}
\xi_{1}\left(P_{n}\right)= & 2 \sum_{i=(n+1) / 2}^{n-1} i^{2}+\left(\frac{n-1}{2}\right)^{2}=2\left(\sum_{i=1}^{n-1} i^{2}-\sum_{i=1}^{(n-1) / 2} i^{2}\right)+\left(\frac{n-1}{2}\right)^{2} \\
= & \frac{1}{3}(n-1) n(2 n-1)-\frac{1}{3} \cdot \frac{(n-1)}{2} \cdot \frac{(n+1)}{2} \cdot n+\left(\frac{n-1}{2}\right)^{2} \\
= & \frac{7 n^{3}-9 n^{2}-n+3}{12}, \\
\xi_{2}\left(P_{n}\right)= & 2 \sum_{i=(n-1) / 2}^{n-2} i(i+1)=2\left(\sum_{i=1}^{n-2} i^{2}-\sum_{i=1}^{(n-3) / 2} i^{2}+\sum_{i=(n-1) / 2}^{n-2} i\right) \\
= & \frac{1}{3}(n-2)(n-1)(2 n-3)-\frac{1}{3} \cdot \frac{(n-3)}{2} \cdot \frac{(n-1)}{2} \cdot(n-2)+ \\
& \frac{(n-1)}{2} \cdot \frac{(3 n-5)}{2} \\
= & \frac{7 n^{3}-21 n^{2}+17 n-3}{12} .
\end{aligned}
$$

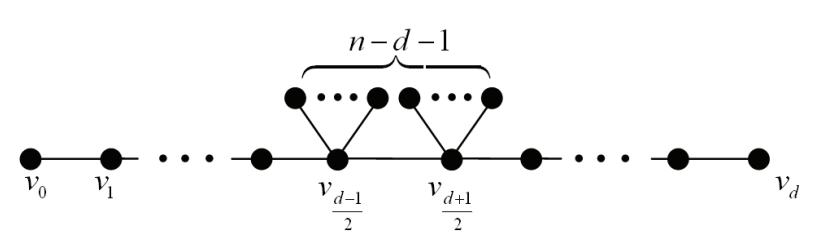

Figure 2. The structure of trees in $\mathrm{L}_{n, d}$ with odd $d$.

For a graph $G$ and a subset $E^{\prime}$ of its edge set ( $E^{*}$ of the edge set of its complement, respectively), $G-E^{\prime}$ $\left(G+E^{*}\right.$, respectively) denotes the graph formed from $G$ by deleting (adding, respectively) edges from $E^{\prime}$ ( $E^{*}$ respectively). For a graph $G$ with $u \in V(G), G-u$ denotes the graph formed from $G$ by deleting the vertex $u$ (and its incident edges).

\section{RESULTS FOR TREES}

In this section, we give lower and upper bounds for the first and the second Zagreb eccentricity indices of $n$-vertex trees with fixed diameter, and lower bounds for the first and the second Zagreb eccentricity indices of $n$ vertex trees with fixed matching number. We also determine the $n$-vertex trees with, respectively, the minimum, second-minimum and third-minimum, as well as the maximum, second-maximum and third-maximum indices $\xi_{1}$ and $\xi_{2}$ for $n \geq 6$.

Lemma 1. Let $u$ be a vertex of a tree $Q$ with at least two vertices. For integer $a \geq 1$, let $G_{1}$ be the tree obtained from $Q$ by attaching a star $S_{a+1}$ at its center $v$ to $u, G_{2}$ the tree obtained from $Q$ by attaching $a+1$ pendant vertices to $u$, see Figure 1 . Then $\xi_{1}\left(G_{2}\right)<$ $\xi_{1}\left(G_{1}\right)$ and $\xi_{2}\left(G_{2}\right)<\xi_{2}\left(G_{1}\right)$.

Let $\mathbf{T}(n, d)$ be the set of $n$-vertex trees with diameter $d$, where $2 \leq d \leq n-1$. For $2 \leq d \leq n-2$, let $\mathrm{L}_{n, d}$ be the set of $n$-vertex trees obtained from $P_{d+1}=v_{0} v_{1} \cdots v_{d}$ by attaching $n-d-1$ pendant vertices to $v_{\lfloor d / 2\rfloor}$ and/or $v_{\lceil d / 2\rceil}$. Let $\mathrm{L}_{n, n-1}=\left\{P_{n}\right\}$. Note that in $\mathrm{L}_{n, d}$, there is only one tree for even $d$, and $\left\lfloor\frac{n-d+1}{2}\right\rfloor$ trees for odd $d$. Let $U_{n, d}^{a}$ be the $n$-vertex tree obtained by attaching $a$ and $n+1-a-d$ pendant vertices, respectively, to the two end vertices of the path $P_{d-1}$ for $1 \leq a \leq\left\lfloor\frac{n+1-d}{2}\right\rfloor$, and let $\mathrm{U}_{n, d}=\left\{U_{n, d}^{a}: 1 \leq a \leq\right.$ $\left\lfloor\frac{n+1-d}{2}\right\rfloor$. See Figures 2-4 for the trees in $\mathrm{L}_{n, d}$ and the tree $U_{n, d}^{a}$.

Proposition 1. Let $T \in \mathbf{T}(n, d)$, where $2 \leq d \leq$ $n-1$. Then: 


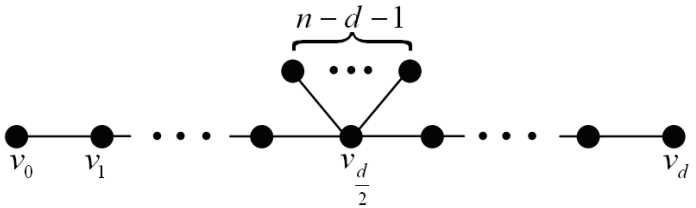

Figure 3. The tree in $\mathrm{L}_{n, d}$ with even $d$.

$$
\xi_{1}(T) \geq \begin{cases}\frac{3(d+2)^{2} n+4 d^{3}-3 d^{2}-22 d-12}{12} & \text { if } d \text { is even } \\ \frac{3(d+3)^{2} n+4 d^{3}-9 d^{2}-40 d-27}{12} & \text { if } d \text { is odd }\end{cases}
$$

$\xi_{2}(T) \geq \begin{cases}\frac{3 d(d+2) n+4 d^{3}-9 d^{2}-10 d}{12} & \text { if } d \text { is even } \\ \frac{3(d+1)(d+3) n+4 d^{3}-15 d^{2}-22 d-3}{12} & \text { if } d \text { is odd }\end{cases}$

with either equality if and only if $T \in \mathrm{L}_{n, d}$.

Proposition 2. Let $T \in \mathbf{T}(n, d)$, where $2 \leq d \leq$ $n-1$. Then:

$$
\begin{gathered}
\xi_{1}(T) \leq \begin{cases}d^{2} n-\frac{5 d^{3}-2 d}{12} & \text { if } d \text { is even } \\
d^{2} n-\frac{5 d^{3}-5 d}{12} & \text { if } d \text { is odd, }\end{cases} \\
\xi_{2}(T) \leq \begin{cases}\left(d^{2}-d\right) n-\frac{5 d^{3}-8 d}{12} & \text { if } d \text { is even } \\
\left(d^{2}-d\right) n-\frac{5 d^{3}-11 d-6}{12} & \text { if } d \text { is odd }\end{cases}
\end{gathered}
$$

with either equality if and only if $T \in \mathrm{U}_{n, d}$.

Now we consider the $n$-vertex trees with fixed matching number. A matching $M$ of the graph $G$ is a subset of $E(G)$ such that no two edges in $M$ share a common vertex. A matching $M$ of $G$ is said to be maximum, if for any other matching $M_{1}$ of $G$, $\left|M_{1}\right| \leq|M|$. The matching number of $G$ is the number of edges of a maximum matching in $G$. If $M$ is a matching of a graph $G$ and vertex $v \in V(G)$ is incident with an edge of $M$, then $v$ is said to be $M$ saturated, and if every vertex of $G$ is $M$-saturated, then $M$ is a perfect matching. For integers $n$ and $1 \leq m \leq\lfloor n / 2\rfloor$, let $\mathbf{T}_{(n, m)}$ be the set of $n$-vertex trees

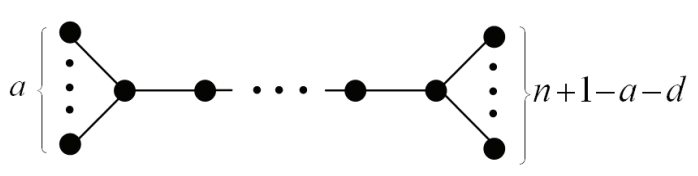

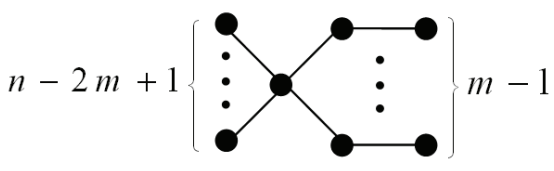

Figure 5. The tree $T_{n, m}$.

with matching number $m$. Obviously, $\mathbf{T}_{(n, 1)}=\left\{S_{n}\right\}$. For $3 \leq m \leq\lfloor n / 2\rfloor$, let $T_{n, m}$ be the tree obtained by attaching $m-1$ paths on two vertices to the center of the star $S_{n-2 m+2}$, see Figure 5 .

Proposition 3. Let $T \in \mathbf{T}_{(2 m, m)}$ with $m \geq 3$. Then $\xi_{1}(T) \geq 25 m-12, \xi_{2}(T) \geq 18 m-12$ with either equality if and only if $T=T_{2 m, m}$.

Proposition 4. Let $T \in \mathbf{T}_{(n, m)}$ with $2 \leq m \leq|n / 2|$. If $m=2$, then $\xi_{1}(T) \geq 9 n-10$ and $\xi_{2}(T) \geq 6 n-8$ with either equality if and only if $T \in \mathbf{T}(n, 3)$;

If $m \geq 3$, then $\xi_{1}(T) \geq 9 n+7 m-12$ and $\xi_{2}(T) \geq 6 n+$ $6 m-12$ with either equality if and only if $T=T_{n, m}$.

Now we use the result for the first and the second Zagreb eccentricity indices of the $n$-vertex trees with fixed diameter to determine the $n$-vertex trees with small and large indices $\xi_{1}$ and $\xi_{2}$.

Proposition 5. Among the $n$-vertex trees, $S_{n}$ for $n \geq 3$, the trees in $\mathrm{L}_{n, 3}$ for $n \geq 4$, and the trees in $\mathrm{L}_{n, 4}$ for $n \geq 5$ are, respectively, the unique trees with the minimum, second-minimum and third-minimum indices $\xi_{1}$ and $\xi_{2}$, the first Zagreb eccentricity indices of which are equal to $4 n-3,9 n-10$ and $9 n+9$, respectively, while the second Zagreb eccentricity indices of which are equal to $2 n-2,6 n-8$ and $6 n+6$, respectively.

For $n \geq 4$, let $P_{n}^{\prime}$ be the tree formed by attaching a pendant vertex to the neighbor of an end-vertex of the path $P_{n-1}$. For $n \geq 6$, let $P_{n}^{\prime \prime}$ be the tree formed by attaching a pendant vertex to the second neighbor of an end-vertex of the path $P_{n-1}$.

Proposition 6. Among the $n$-vertex trees, $P_{n}$ for $n \geq 3, \quad P_{n}^{\prime}$ for $n \geq 4$, and $P_{n}^{\prime \prime}$ for $n \geq 5$ are, respectively, the unique trees with the maximum, secondmaximum and third-maximum indices $\xi_{1}$ and $\xi_{2}$, where:

$$
\xi_{1}\left(P_{n}\right)= \begin{cases}\frac{7 n^{3}-9 n^{2}+2 n}{12} & \text { if } n \text { is even } \\ \frac{7 n^{3}-9 n^{2}-n+3}{12} & \text { if } n \text { is odd }\end{cases}
$$

Figure 4. The tree $U_{n, d}^{a}$. 


$$
\begin{aligned}
& \xi_{2}\left(P_{n}\right)= \begin{cases}\frac{7 n^{3}-21 n^{2}+20 n}{12} & \text { if } n \text { is even } \\
\frac{7 n^{3}-21 n^{2}+17 n-3}{12} & \text { if } n \text { is odd, }\end{cases} \\
& \xi_{1}\left(P_{n}^{\prime}\right)= \begin{cases}\frac{7 n^{3}-18 n^{2}-10 n+36}{12} & \text { if } n \text { is even } \\
\frac{7 n^{3}-18 n^{2}-7 n+30}{12} & \text { if } n \text { is odd, }\end{cases} \\
& \xi_{2}\left(P_{n}^{\prime}\right)= \begin{cases}\frac{7 n^{3}-30 n^{2}+20 n+24}{12} & \text { if } n \text { is even } \\
\frac{7 n^{3}-30 n^{2}+23 n+24}{12} & \text { if } n \text { is odd, }\end{cases} \\
& \xi_{2}\left(P_{n}^{\prime \prime}\right)= \begin{cases}\frac{7 n^{3}-30 n^{2}-4 n+96}{12} & \text { if } n \text { is even } \\
\frac{7 n^{3}-30 n^{2}-n+96}{12} & \text { if } n \text { is odd. }\end{cases} \\
& \xi_{1}\left(P_{n}^{\prime \prime}\right)= \begin{cases}\frac{7 n^{3}-18 n^{2}-34 n+96}{12} & \text { if } n \text { is even } \\
\frac{7 n^{3}-18 n^{2}-31 n+90}{12} & \text { is odd, }\end{cases}
\end{aligned}
$$

\section{RESULTS FOR GENERAL CONNECTED GRAPHS}

In this section, we give lower and upper bounds for the first and the second Zagreb eccentricity indices of connected graphs in terms of graph invariants such as the number of vertices, the number of edges, the radius and the diameter, and we also come up with the NordhausGaddum-type results. ${ }^{14}$ Moreover, among the $n$-vertex connected graphs, we establish lower and upper bounds for the first Zagreb eccentricity index, and lower bound for the second Zagreb eccentricity index, respectively, and characterize the extremal cases.

Proposition 7. Let $G$ be a connected graph with $n$ vertices and $m$ edges. Then:

$$
\begin{aligned}
n r(G)^{2} & \leq \xi_{1}(G) \leq n D(G)^{2}, \\
m r(G)^{2} & \leq \xi_{2}(G) \leq m D(G)^{2}
\end{aligned}
$$

with any equality if and only if $G$ is a self-centered graph.

Proposition 8. Let $G$ be a connected graph with $n \geq 2$ vertices. Then:

$$
\xi_{1}\left(K_{n}\right) \leq \xi_{1}(G) \leq \xi_{1}\left(P_{n}\right)
$$

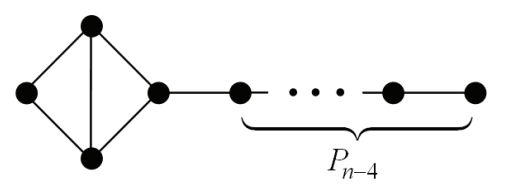

Figure 6. The graph $B_{n}$.

with left equality if and only if $G=K_{n}$ and right equality if and only if $G=P_{n}$.

Corollary 1. Let $G$ be a connected bipartite graph with $n \geq 3$ vertices. Then $\xi_{1}(G) \geq 4 n-3$ with equality if and only if $G=S_{n}$.

Proposition 9. Let $G$ be a connected graph with $n \geq 2$ vertices. Then:

$$
\xi_{2}(G) \geq \begin{cases}1 & \text { if } n=2 \\ 3 & \text { if } n=3 \\ 2(n-1) & \text { if } n \geq 4\end{cases}
$$

with equality if and only if $G=K_{n}$ for $n=2,3$, $G=K_{4}$ or $S_{4}$ for $n=4$, and $G=S_{n}$ for $n \geq 5$.

Remark. By Proposition 8, among the $n$-vertex connected graphs, $P_{n}$ is the unique graph with the maximum first Zagreb eccentricity index. However, $P_{n}$ is not the graph with the maximum second Zagreb eccentricity index for $n \geq 9$. For $n \geq 5$, let $B_{n}$ be the bicyclic graph obtained by attaching a path $P_{n-4}$ (at one end vertex) to a vertex of degree two in the bicyclic graph obtained by adding an edge to the quadrangle, see Figure 6. Then:

$$
\begin{aligned}
\xi_{2}\left(B_{n}\right)= & \xi_{2}\left(P_{n-1}\right)+(n-2)(n-3)+(n-3)^{2}+(n-3)(n-4) \\
& = \begin{cases}\frac{7 n^{3}-6 n^{2}-136 n+276}{12} & \text { if } n \text { is even } \\
\frac{7 n^{3}-6 n^{2}-133 n+276}{12} & \text { if } n \text { is odd. }\end{cases}
\end{aligned}
$$

For $n \geq 9$ :

$\xi_{2}\left(B_{n}\right)-\xi_{2}\left(P_{n}\right)=$
$= \begin{cases}\frac{7 n^{3}-6 n^{2}-136 n+276}{12}-\frac{7 n^{3}-21 n^{2}+20 n}{12} & \text { if } n \text { is even } \\ \frac{7 n^{3}-6 n^{2}-133 n+276}{12}-\frac{7 n^{3}-21 n^{2}+17 n-3}{12} & \text { if } n \text { is odd }\end{cases}$

$= \begin{cases}\frac{15 n^{2}-156 n+276}{12} & \text { if } n \text { is even } \\ \frac{15 n^{2}-150 n+279}{12} & \text { if } n \text { is odd }\end{cases}$

$>0$,

and then $\xi_{2}\left(B_{n}\right)>\xi_{2}\left(P_{n}\right)$. 
Denote by $\bar{G}$ the complement of a graph $G$. There is only one connected graph $P_{4}$ on four vertices with the connected complement $\overline{P_{4}}=P_{4}$. Obviously, we have $\xi_{1}\left(P_{4}\right)+\xi_{1}\left(\overline{P_{4}}\right)=2 \xi_{1}\left(P_{4}\right)=52$. For $n \geq 5$, the diameter of $P_{n}$ is two.

Now we give the Nordhaus-Gaddum-type results.

Proposition 10. Let $G$ be a connected graph with $n \geq 4$ vertices for which $\bar{G}$ is also connected. Then:

$$
8 n \leq \xi_{1}(G)+\xi_{1}(\bar{G}) \leq \xi_{1}\left(P_{n}\right)+\xi_{1}\left(\overline{P_{n}}\right)
$$

with left equality if and only if $G$ and $\bar{G}$ are selfcentered graphs with radius two and right equality if and only if $G=P_{n}$ or $\overline{P_{n}}$, and $\xi_{2}(G) \pm \xi_{2}(\bar{G}) \geq 2 n(n-1)$ with equality if and only if $G$ and $\bar{G}$ are self-centered graphs with radius two.

\section{CONCLUSION}

In this paper, we study the novel molecular descriptors, called Zagreb eccentricity indices, which were proposed analogously to Zagreb indices already known and used for almost forty years. We give lower and upper bounds for Zagreb eccentricity indices of trees and general graphs in terms of graph invariants, and characterize the extremal graphs, and determine the $n$-vertex trees with small and large Zagreb eccentricity indices. We also give Nordhaus-Gaddum-type results for Zagreb eccentricity indices. The lower and upper bounds for a molecular descriptor in terms of some graph invariants (e.g., the number of vertices) are important information for a molecule in the sense that they establish the range of the descriptor.

Supplementary Materials. - Supporting informations to the paper are enclosed to the electronic version of the article.
These data can be found on the website of Croatica Chemica Acta (http://public.carnet.hr/ccacaa).

Acknowledgements. This work is supported by the National Natural Science Foundation of China (Grant No. 11071089) and the Ministry of Science, Education and Sports of Croatia (Grant No. 098-1770945-2919).

\section{REFERENCES}

1. F. Harary, Graph Theory, 2nd printing, Addison-Wesley, Reading, MA, 1971, p. 35.

2. I. Gutman and N. Trinajstić, Chem. Phys. Lett. 17 (1972) 535538.

3. I. Gutman, B. Ruščić, N. Trinajstić, and C.F. Wilcox, Jr., J. Chem. Phys. 62 (1975) 3399-3405.

4. S. Nikolić, G. Kovačević, A. Miličević, and N. Trinajstić, Croat. Chem. Acta 76 (2003) 113-124.

5. D. Vukičević and A. Graovac, Acta Chim. Slov. 57 (2010) 524-538.

6. R. Todeschini and V. Consonni, Handbook of Molecular Descriptors, Wiley-VCH, Weinheim, 2000, p. 124.

7. R. Todeschini and V. Consonni, Molecular Descriptors for Chemoinformatics, Vol. I, Wiley-VCH, Weinheim, 2009, pp. 237-241.

8. V. Sharma, R. Goswami, and A. K. Madan, J. Chem. Inf. Comput. Sci. 37 (1997) 273-282.

9. S. Sardana and A. K. Madan, J. Comput. Aid. Mol. Des. 16 (2002) 545-550

10. B. Zhou and Z. Du, MATCH Commun. Math. Comput. Chem. 63 (2010) 181-198.

11. A. Ilić, In: I. Gutman and B. Furtula (Eds.), Novel Molecular Structure Descriptors - Theory and Applications II, Univ. Kragujevac, Kragujevac, 2010, pp. 139-168.

12. M. Saheli and A. R. Ashrafi, J. Comput. Theor. Nanosci. 7 (2010) 1900-1903

13. S. Gupta, M. Singh, and A. K. Madan, J. Comput. Aid. Mol. Des. 15 (2001) 671-678.

14. E. A. Nordhaus and J. W. Gaddum, Amer. Math. Mon. 63 (1956) 175-177.

15. R. A. Brualdi and J. L. Goldwasser, Discr. Math. 48 (1984) 1-21. 